\title{
miR-221-3p and miR-15b-5p promote cell proliferation and invasion by targeting Axin2 in liver cancer
}

\author{
YEFENG DONG $^{1 *}$, NANNAN ZHANG $^{2 *}$, SUMING ZHAO $^{3}$, XIAOYANG CHEN $^{4},{\text { FENG } \mathrm{LI}^{5} \text { and XIA TAO }}^{6}$ \\ ${ }^{1}$ Department of Clinical Laboratory, The Affiliated Haian Hospital of Nantong University, Nantong, Jiangsu 226001; \\ ${ }^{2}$ Department of General Surgery, Tongzhou People's Hospital, Nantong, Jiangsu 226399; \\ Departments of ${ }^{3}$ Tumor Intervention, ${ }^{4}$ Medical Ultrasound, ${ }^{5}$ Gastroenterology and \\ ${ }^{6}$ Pharmacy, The Affiliated Hospital of Nantong University, Nantong, Jiangsu 226001, P.R. China
}

Received May 4, 2019; Accepted October 11, 2019

DOI: $10.3892 / \mathrm{ol} .2019 .11056$

\begin{abstract}
Globally, liver cancer has the third highest mortality rate among all types of cancer due to the invasive and metastatic capacities of liver tumor cells. MicroRNA (miR) is a class of non-coding RNAs that participate in the development of liver cancer. The aim of the present study was to explore the molecular mechanisms by which miR-221-3p and miR-15b-5p promote the proliferation and invasion of liver cancer cells through targeting axis formation inhibitor 2 (Axin2) and to identify suitable targets for the treatment of liver cancer. The expression levels of miR-221-3p and miR-15b-5p were determined in liver cancer tissues and cells by quantitative PCR, and the association between miR-221-3p, miR-15b-5p and Axin 2 expression in liver cancer cells was analyzed using cell transfection. The results demonstrated that miR-221-3p and miR-15b-5p levels were upregulated in liver cancer tissues and cell lines, and results from predictive bioinformatic analysis and identification revealed that Axin 2 was the common target gene of miR-221-3p and miR-15b-5p. miR-221-3p and miR-15b-5p may be used as prognostic indicators for liver cancer. The miR-221-3p/miR-15b-5p-Axin2 axis may serve as a therapeutic target in patients with liver cancer.
\end{abstract}

\section{Introduction}

Primary liver cancer is a malignant tumor with the third highest mortality rate in the world (1). The long-term survival remains unsatisfactory due to high incidence of recurrence and metastasis following hepatic resection (2). Due to the

Correspondence to: Dr Xia Tao, Department of Pharmacy, The Affiliated Hospital of Nantong University, Nantong, Jiangsu 226001, P.R. China

E-mail: xiatao79@yeah.net

*Contributed equally

Key words: liver cancer, miR-221-3p, miR-15b-5p, Axin2, proliferation, therapeutic target large number of genes and proteins involved, the pathogenesis of liver cancer is particularly complicated (3-5). Therefore, revealing the molecular mechanism of liver cancer pathogenesis is key to the development of effective treatment.

MicroRNAs (miRNAs) are a class of endogenous highly evolutionarily conserved single-stranded non-coding RNAs with a total length of 18-25 nucleotides. As regulators of negative regulatory genes, miRNAs are completely or incompletely complementary to the 3'untranslated regions (3'-UTRs) of their target genes, which results in direct degradation or translational disruption of these genes (6). Ji et al (7) have demonstrated that all members of the miRNA (miR)-181 family were upregulated in epithelial cell adhesion molecule (EpCAM/CD326)-positive hepatic cancer stem cells isolated from $\alpha$-fetoprotein (AFP)-positive liver cancer samples. Tomimaru et al (8) compared serum miR-21 levels in 126 cases of liver cancer, 30 cases of chronic hepatitis and 50 healthy volunteers; the results revealed the level of miR-21 expression to be significantly higher in patients with liver cancer compared with those with chronic hepatitis and healthy volunteers. Abnormal expression of miR-221 has been observed in multiple types of cancer, such as liver (9), gastric (10) and breast (11) cancer, suggesting that it may be closely associated with tumorigenesis and may provide a novel target for tumor diagnosis and treatment. miR-15b dysregulation has also been reported in various types of cancer. For example, the $\mathrm{miR}-15 \mathrm{~b} / \mathrm{HOTAIR} / \mathrm{p} 53$ regulatory loop affects glioma cell proliferation (12). miR-15b promotes prostate cancer cell proliferation by targeting reversion-inducing cysteine-rich protein with Kazal motifs and may also be used as a clinical diagnostic marker in patients with prostate cancer (13).

The present study aimed to analyze miR-221-3p and miR-15b-5p expression in liver cancer to establish their potential roles and target genes.

\section{Materials and methods}

Patients and samples. A total of 69 patients with liver cancer (40 men and 29 women; mean age 58.3 \pm 5.9 years) who underwent surgical resection at Affiliated Hospital of Nantong University between October 2010 and May 2012 were recruited for the present study. Tumor and adjacent 
non-cancerous tissues ( $>2 \mathrm{~cm}$ from the lesion) were collected and stored at $-80^{\circ} \mathrm{C}$ until further use. The patients did not receive chemotherapy, radiotherapy, biological therapy or a similar treatment regimen prior to surgery. The pathological classification and staging of liver cancer were consistent with the 7th edition of the American Joint Committee on Cancer Tumor-Node-Metastasis (TNM) Staging System published in 2010 (14). This study was approved by the Ethics Committee of The Affiliated Hospital of Nantong University and written informed consent was obtained from all patients.

Cell culture and transfection. Human liver cancer cell lines HepG2 and Huh7 were purchased from the American Type Culture Collection, and Hep3B and HCCLM3 cell lines were purchased from the type Culture Collection of the Chinese Academy of Sciences. All cells were authenticated by short tandem repeat profiling. Cells were cultured with DMEM (Invitrogen; Thermo Fisher Scientific, Inc.) containing 10\% FBS (Invitrogen; Thermo Fisher Scientific, Inc.), $100 \mathrm{U} / \mathrm{ml}$ penicillin and $100 \mu \mathrm{g} / \mathrm{ml}$ streptomycin (Sigma-Aldrich; Merck KGaA) and placed at $37^{\circ} \mathrm{C}$ in a humidified incubator containing $5 \% \mathrm{CO}_{2}$. Vectors expressing AXIN2, AXIN2 small interfering RNA (siRNA) (5'-3') and scrambled control-sense ( $\left.5^{\prime}-3^{\prime}\right)$ were designed and synthesized by Chang Jing Bio-Tech, Ltd. Lipofectamine ${ }^{\circledR} 3000$ Transfection Reagent (Invitrogen; Thermo Fisher Scientific, Inc.) was used for cell transfections according to the manufacturer's instructions. The miRNA mimics and inhibitor and AXIN2 siRNA were purchased from Sangon Biotech Co., Ltd. The pcDNA3.1A(-) vector and pcDNA3.1A(-)-AXIN2 overexpression plasmid were obtained from Shanghai GeneChem Co., Ltd. The sequences were as follows: miR-221-3p mimics, 5'-AGCUACAUUGUC UGCUGGGUUUC-3', 5'-AACCCAGCAGACAAUGUAGCU UU-3'; miR-221-3p inhibitor, 5'-GAAACCCAGCAGACA AUGUAGCU-3; miR-15b-5p inhibitor, 5'-UGUAAACCAUGA UGUGCUGCUA-3'; miR-15b-5p mimics: 5'-UAGCAGCAC AUCAUGGUUUACA-3', 5'-UAAACCAUGAUGUGCUGC UAUU-3'; and negative control, 5'-UUCUCCGAACGUGUC ACGUTT-3' and 5'-ACGUGACACGUUCGGAGAATT-3'. The sequences of siRNA and its negative control were as follows: Axin2, 5'-GCAGAGGGACAGGAATCAT-3', and the negative control, 5'-GCAGGGACAAGGTAGACAT-3'.

Transfection of liver cancer cells was performed using Lipofectamine ${ }^{\circledR} 3000$ (Invitrogen; Thermo Fisher Scientific, Inc.). Transfection efficiency was verified by western blotting $48 \mathrm{~h}$ post-transfection. For functional analysis of miR-221-3p and miR-15b-5p, liver cancer cells were transfected with miR-221-3p and miR-15b-5p mimics and the control (miR-con) or miR-221-3p and miR-15b-5p inhibitor and negative control (scramble).

RNA extraction and quantitative (q)PCR. According to manufacturer's instructions, total RNA was extracted by TRIzol ${ }^{\circledR}$ (Invitrogen; Thermo Fisher Scientific, Inc.) from tissues or liver cancer cells, including Hep3B, HepG2, HCCLM3 and Huh7 cell lines. Reverse transcription reaction was performed using SYBR Premix Ex Taq (Takara Bio, Inc.) at $16^{\circ} \mathrm{C}$ for $30 \mathrm{~min}$, $42^{\circ} \mathrm{C}$ for $30 \mathrm{~min}$ and $85^{\circ} \mathrm{C}$ for $5 \mathrm{~min}$. qPCR was performed using SYBR Premix Ex Taq with $1 \mu \mathrm{l}$ cDNA from the RT reaction on an ABI 7500 system (Applied Biosystems; Thermo
Fisher Scientific, Inc.). The PCR thermocycling conditions were as follows: $94^{\circ} \mathrm{C}$ for $2 \mathrm{~min}$ followed by 30 cycles of $94^{\circ} \mathrm{C}$ for $30 \mathrm{sec}, 58^{\circ} \mathrm{C}$ for $30 \mathrm{sec}$, and $72^{\circ} \mathrm{C}$ for $30 \mathrm{sec}$. U6 small RNA was used as an internal reference. Primers were synthesized by Sangon Biotech Co., Ltd. as follows: miR-221-3p, forward, 5'-CGGCTACATTGTCTGCCTG-3' and reverse, 5'-CAGTGC GTGTCGTGGAGT-3'; miR-15b-5p forward, 5'-ATGAAC TTTCTCTGTCTTGG-3' and reverse, 5'-CAGTGCGTGTCG TGGAGT-3'; and U6 forward, 5'-CGCTTCGGCAGCACA TATAC-3' and reverse, 5'-AACGCTTCACGAATTTGCGT-3'. The reverse universal miR qPCR primers were included in the PrimeScript $^{\mathrm{TM}}$ miRNA RT-PCR kit (cat. no. RR716; Takara Biotechnology Co., Ltd.). The relative expression levels of miR-221-3p and miR-15b-5p were calculated using the $2^{-\Delta \Delta C q}$ method as previously described (15). All experiments were conducted in triplicate.

Cell viability assay. Cell viability assay was performed using the 3-(4,5-dimethylthiazole-2-yl)-2,5-biphenyl tetrazolium bromide (MTT) method with DMSO as solvent $(3,000$ cells per well in 96-well plates). Absorbance was detected at $570 \mathrm{~nm}$ in three parallel samples, and each sample was tested three times.

Colony formation assay. At $24 \mathrm{~h}$ post-transfection, cells were seeded into 6-well plates and cultured for two weeks in DMEM containing $12 \% \mathrm{FBS}$ at $37^{\circ} \mathrm{C}$ in a humiliated atmosphere of $5 \% \mathrm{CO}_{2}$ (600 cells per well in 6-well plates). The colonies were fixed and stained with $1 \mathrm{mg} / \mathrm{ml}$ crystal violet, and colonies containing $>50$ cells were counted using Leica microscopy (Leica DMR 3000; Leica Microsystems GmbH).

Transwell assay. Cell invasive ability was determined using a Transwell assay with Matrigel (BD Biosciences). To assess cell invasion, Transwell chambers were coated with $30 \mu \mathrm{l}$ Matrigel, incubated at $37^{\circ} \mathrm{C}$ for $40 \mathrm{~min}$ and placed in 24-well plates. At 24 h post-transfection, HepG 2 and HCCLM3 cells were seeded in the upper chamber at a density of $5 \times 10^{4}$ cells/well in DMEM with $2 \%$ FBS. A total of $500 \mu 1$ DMEM containing $10 \% \mathrm{FBS}$ was added to the lower chamber. After $24 \mathrm{~h}$ at $37^{\circ} \mathrm{C}$, invaded cells were fixed with $4 \%$ paraformaldehyde for $10 \mathrm{~min}$ at $37^{\circ} \mathrm{C}$. Non-invaded cells were removed with a cotton swab. Invaded cells were stained with $1 \mathrm{mg} / \mathrm{ml}$ crystal violet at room temperature for $10 \mathrm{~min}$ and counted in five randomly selected fields using Leica microscope (Leica DMR 3000; Leica Microsystems GmbH).

Dual luciferase reporter assay. The 3' UTR of Axin2 was cloned into a pGL3 control vector (Promega Corporation) containing luciferase genes to generate pGL3-AXIN2-wild-type plasmids. The primers are as follows: Axin2 forward, 5'-GCT CTAGAGCCCTGGGGTCTGGCTTTG-3' and reverse, 5'-GCTCTAGAT TTT GAA AAATATAAA ATT-3'. The pGL3-AXIN2-mutant plasmids were constructed using a Takara MutanBEST Kit (Takara Biotechnology Co., Ltd.). HCCLM3 and HepG2 cells were seeded into 24-well plates $\left(1 \times 10^{5}\right.$ cells/well), cultured for $24 \mathrm{~h}$ at $37^{\circ} \mathrm{C}$ and transfected with the miRNA mimic or inhibitor and the corresponding luciferase reporter plasmids using Lipofectamine ${ }^{\circledR} 3000$. pRL-TK Renilla plasmids were co-transfected into the cells as an internal reference. At $48 \mathrm{~h}$, the reporter activity 

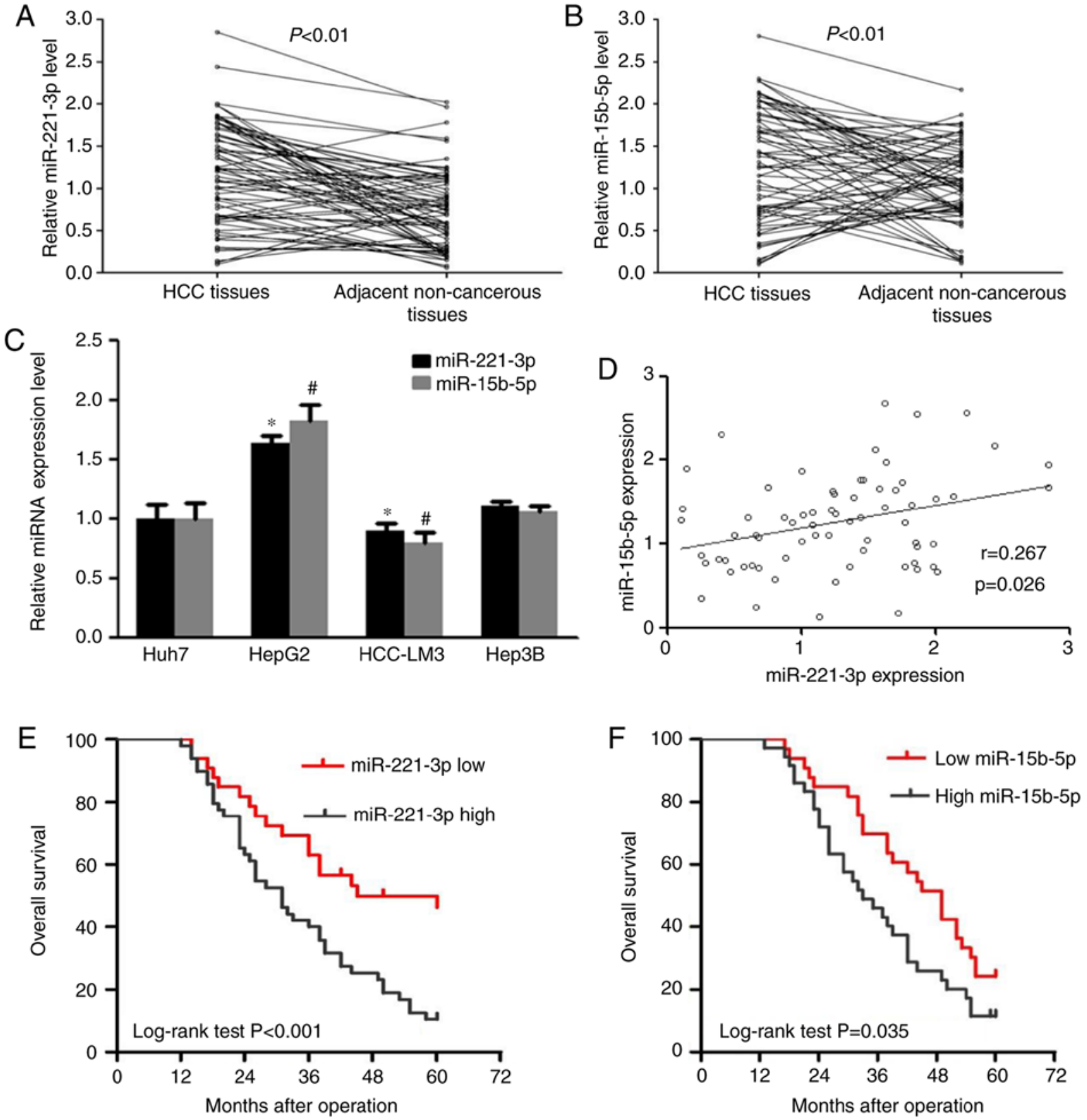

Figure 1. miR-221-3p and miR-15b-5p are significantly upregulated in liver cancer tissues and cell lines. (A) Relative miR-221-3p expression levels (1.85 \pm 1.19$)$ in liver cancer and adjacent non-cancerous tissues $(1.02 \pm 0.39)(\mathrm{N}=69)$. The value was represented by mean $\pm \mathrm{SD}$. (B) Relative miR-15b-5p expression levels in liver cancer and adjacent non-cancerous tissues ( $\mathrm{N}=69$ ). (C) Relative miR-221-3p and miR-15b-5p expression levels in liver cancer cell lines. (D) Correlation between miR-221-3p and miR-15b-5p expression in liver cancer tissues. (E) Kaplan-Meier analysis of the overall survival of patients with liver cancer with high $(\mathrm{N}=36)$ and low $(\mathrm{N}=33)$ miR-221-3p expression levels. (F) Kaplan-Meier analysis of the overall survival of patients with liver cancer with high ( $\mathrm{N}=36)$ and low (N=33) miR-15b-5p expression levels. " $\mathrm{P}<0.05$ and ${ }^{~} \mathrm{P}<0.05$ vs. Huh7. miR, miRNA, microRNA; HCC, hepatocellular carcinoma.

was measured using a Dual Luciferase Reporter Assay Kit (Promega Corporation), and relative to Renilla luciferase activities were determined. The online target gene prediction software packages TargetScan 7.2 (http://www.targetscan.org) and miRanda 3.2 (http://www.mirdb.org) were used.

Western blotting. Total protein of HepG2 cells was extracted according to the manufacturer's instructions using RIPA lysis buffer containing protease inhibitors (Promega Corporation). Protein concentration was determined using bicinchoninic acid assay kit (Bio-Rad Laboratories, Inc.). Proteins (30 $\mu \mathrm{g}$ per lane) were separated using $12 \%$ SDS-PAGE and transferred onto a polyvinylidene fluoride membrane. Following blocking with 5\% skimmed milk in TBS at room temperature for $2 \mathrm{~h}$, the membrane was incubated with rabbit anti-Axin2 polyclonal antibody (1:1,000; cat. no. ab32197; Abcam), rabbit anti- $\beta$-actin polyclonal antibody (1:5,000; cat. no. ab8227; Abcam) and rabbit anti-GAPDH polyclonal antibody (1:5,000; cat. no. ab37168; Abcam) for $12 \mathrm{~h}$ at $4^{\circ} \mathrm{C}$. The membrane was washed three times in TBS + Tween-20 $(0.1 \% \mathrm{~V} / \mathrm{V})$ and incubated with a horseradish peroxidase-conjugated goat anti-rabbit secondary antibody (1:5,000; Sigma-Aldrich; Merck KGaA) at room temperature for $2 \mathrm{~h}$ bands were detected using enhanced chemiluminescence substrate, and relative expression of proteins was normalized to GAPDH using Scion Image v. 4.0.2 software (Scion Corporation). 
Table I. Association between miR-221 expression and clinicopathological parameters of patients with liver cancer.

\begin{tabular}{|c|c|c|c|c|c|}
\hline $\begin{array}{l}\text { Clinicopathological } \\
\text { characteristic }\end{array}$ & $\mathrm{N}$ & $\begin{array}{c}\text { Relative } \\
\text { miR-221-3p expression }\end{array}$ & P-value & $\begin{array}{c}\text { Relative } \\
\text { miR-15b-5p expression }\end{array}$ & P-value \\
\hline Sex & & & 0.464 & & 0.431 \\
\hline Male & 40 & $1.99 \pm 1.68$ & & $1.85 \pm 1.48$ & \\
\hline Female & 29 & $1.73 \pm 1.29$ & & $1.64 \pm 1.33$ & \\
\hline Age, years & & & 0.231 & & 0.325 \\
\hline$\geq 60$ & 35 & $1.96 \pm 1.71$ & & $1.68 \pm 1.51$ & \\
\hline$<60$ & 34 & $1.37 \pm 0.85$ & & $1.48 \pm 0.92$ & \\
\hline Tumor size, $\mathrm{cm}$ & & & 0.169 & & 0.201 \\
\hline$\geq 5$ & 36 & $2.32 \pm 1.41$ & & $1.98 \pm 1.68$ & \\
\hline$<5$ & 33 & $1.73 \pm 0.58$ & & $1.69 \pm 0.98$ & \\
\hline AFP, ng/ml & & & 0.651 & & 0.520 \\
\hline$<400$ & 27 & $2.11 \pm 0.83$ & & $2.01 \pm 1.02$ & \\
\hline$\geq 400$ & 42 & $1.96 \pm 1.15$ & & $1.99 \pm 1.25$ & \\
\hline HBsAg & & & 0.523 & & 0.488 \\
\hline$(+)$ & 41 & $1.71 \pm 1.02$ & & $1.88 \pm 1.23$ & \\
\hline$(-)$ & 28 & $1.68 \pm 0.89$ & & $1.72 \pm 0.96$ & \\
\hline Clinical TNM stage & & & $0.007^{\mathrm{a}}$ & & $0.005^{\mathrm{a}}$ \\
\hline $\mathrm{I}-\mathrm{II}$ & 29 & $0.75 \pm 0.83$ & & $0.82 \pm 0.69$ & \\
\hline III-IV & 40 & $2.14 \pm 0.47$ & & $2.25 \pm 0.85$ & \\
\hline Tumor capsular infiltration & & & $0.011^{\mathrm{a}}$ & & $0.016^{\mathrm{a}}$ \\
\hline No & 29 & $0.88 \pm 1.11$ & & $0.91 \pm 0.90$ & \\
\hline Yes & 40 & $2.77 \pm 1.38$ & & $2.52 \pm 1.42$ & \\
\hline Cirrhosis & & & 0.722 & & 0.689 \\
\hline Yes & 37 & $1.82 \pm 1.41$ & & $1.96 \pm 1.52$ & \\
\hline No & 32 & $1.62 \pm 1.39$ & & $1.79 \pm 1.47$ & \\
\hline Differentiation & & & 0.332 & & 0.433 \\
\hline Poorly differentiated & 31 & $1.91 \pm 1.28$ & & $1.87 \pm 1.48$ & \\
\hline $\begin{array}{l}\text { Moderately/well } \\
\text { differentiated }\end{array}$ & 38 & $2.09 \pm 1.56$ & & $2.15 \pm 1.34$ & \\
\hline
\end{tabular}

${ }^{\text {aP }}<0.05$. AFP, $\alpha$-fetoprotein; HBsAg, hepatitis B virus surface antigen; TNM, Tumor-Node-Metastasis.

Statistical analysis. SPSS 17.0 (SPSS, Inc.) was used for statistical analysis. Data are expressed as the mean \pm standard deviation. Multiple groups were compared using one-way ANOVA followed by the Student-Newman-Keuls test. The Kaplan-Meier method was used to evaluate the survival rate. Spearman's rank analysis was used to identify the correlation between two groups. $\mathrm{P}<0.05$ was considered to indicate a statistically significant difference.

\section{Results}

miR-221-3p and miR-15b-5p are upregulated in liver cancer tissues and cell lines. RT-qPCR was used to determine the relative expression levels of $\mathrm{miR}-221-3 p$ and $\mathrm{miR}-15 \mathrm{~b}-5 \mathrm{p}$ in liver cancer and adjacent non-cancerous tissues. The results demonstrated that the relative expression level of miR-221-3p was $1.85 \pm 1.19$ in liver cancer tissues, which was significantly higher compared with that in adjacent non-cancerous tissues, in which an expression level of $1.02 \pm 0.39$ was observed
$(\mathrm{P}<0.01$; Fig. 1A). miR-15b-5p levels in liver cancer tissues was also significantly higher compared within the adjacent tissues $(\mathrm{P}<0.01$; Fig. 1B). The expression levels of miR-221-3p and $\mathrm{miR}-15 \mathrm{~b}-5 \mathrm{p}$ were also determined in liver cancer cell lines Hep3B, HepG2, HCCLM3 and Huh7 using RT-qPCR. The results indicated that miR-221-3p and miR-15b-5p were expressed at a higher level in HepG2 cells and at a lower level in HCCLM3 cells compared with Huh7 cells $(\mathrm{P}<0.05$; Fig. 1C). Spearman's rank analysis was used to identify the correlation between miR-221-3p and miR-15b-5p expression levels in liver cancer tissues; the results demonstrated that their expression in tumor tissues exhibited a weak correlation $(r=0.267, P=0.026$; Fig. 1D).

miR-221-3p and miR-15b-5p levels are associated with clinicopathological characteristics and prognosis of patients with liver cancer. The clinicopathological characteristics of 69 patients with liver cancer are presented in Table I. The results of statistical Student-Newman-Keuls test demonstrated 

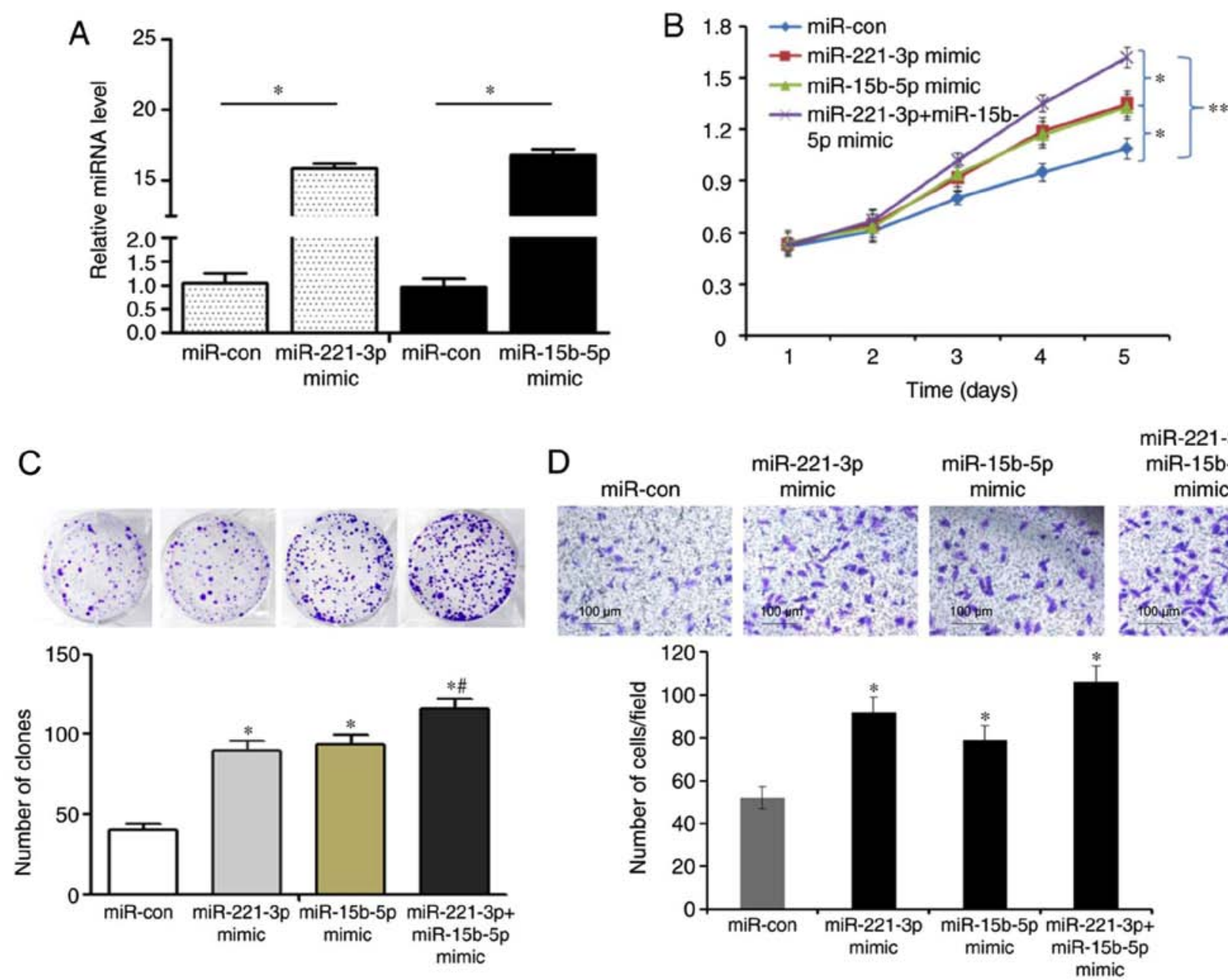

Figure 2. miR-221-3p and/or miR-15b-5p overexpression promotes liver cancer cell proliferation, colony formation and invasion in vitro. (A) Quantitative PCR results demonstrated that miR-221-3p and miR-15b-5p expression levels were significantly increased in HCCLM3 cells transfected with miR-221-3p and miR-15b-5p mimic compared with the control groups. (B) MTT assay results revealed that miR-221-3p and/or miR-15b-5p overexpression significantly increased the viability of HCCLM3 cells compared with the control group. (C) miR-221-3p and/or miR-15b-5p overexpression significantly increased HCCLM3 colony formation. (D) miR-221-3p and/or miR-15b-5p overexpression significantly promoted HCCLM3 cell invasion. ${ }^{*} \mathrm{P}<0.05$ and ${ }^{* *} \mathrm{P}<0.01$ vs. miR-con; ${ }^{\#} \mathrm{P}<0.05$ vs. miR-221-3p + miR-15b-5p mimic. miR, miRNA, microRNA; miR-con, mimic control.

that miR-221-3p and miR-15b-5p levels were associated with the TNM stage and tumor capsular infiltration $(\mathrm{P}<0.05)$, but not with sex, age, tumor size, AFP, hepatitis B virus surface antigen, cirrhosis or tumor differentiation $(\mathrm{P}>0.05)$. Kaplan-Meier analysis revealed that patients with liver cancer with high expression levels of miR-221-3p or miR-15b-5p exhibited lower overall survival rates compared with those with low levels of expression of the respective miRNA $(\mathrm{P}<0.001$ and $\mathrm{P}=0.035$, respectively; Fig. $1 \mathrm{E}$ and $\mathrm{F})$.

Promotive effects of miR-221-3p and miR-15b-5p on liver cancer cell proliferation and invasion in vitro. Based on the analysis of the patient clinicopathological data, it was hypothesized that miR-221-3p and miR-15b-5p may promote liver cancer cell proliferation and invasion. miR-221-3p and miR-15b-5p expression levels were the lowest in HCCLM3 cells, which were selected for overexpression experiments by transfection with miR-221-3p and miR-15b-5p mimics $(\mathrm{P}<0.05$; Fig. 2A). Overexpression of miR-221-3p and/or miR-15b-5p significantly promoted the proliferation of HCCLM3 cells, as indicated by the results of MTT $(\mathrm{P}<0.05$ and $\mathrm{P}<0.01$; Fig. 2B) and colony formation $(\mathrm{P}<0.05 ; \mathrm{Fig} .2 \mathrm{C})$ assays. Overexpression of miR-221-3p and/or miR-15b-5p also stimulated cell invasion $(\mathrm{P}<0.05$; Fig. 2D).

Compared with the other liver cancer cell lines, the relative levels of miR-221-3p and miR-15b-5p were the highest in HepG2 cells, which were selected for transfection with miR-221-3p and miR-15b-5p inhibitors to knock down endogenous miR-221-3p and miR-15b-5p expression $(\mathrm{P}<0.05$, Fig. 3A); knockdown of miR-221-3p and/or miR-15b-5p significantly inhibited cell proliferation, colony formation and invasion $(\mathrm{P}<0.05$; Fig. 3B-D).

Axin2 is a common target of miR-221-3p and miR-15b-5p. To establish the specific mechanism by which miR-221-3p and miR-15b-5p may promote the proliferation of liver cancer cells, the common target gene of miR-221-3p and miR-15b-5p was predicted through bioinformatic analysis using online target gene prediction software packages TargetScan 7.2 and miRanda 3.2 to determine whether the candidate target gene 3'UTR contained miR-221-3p and/or miR-15b-5p binding sites (Fig. 4A and B). The results of 

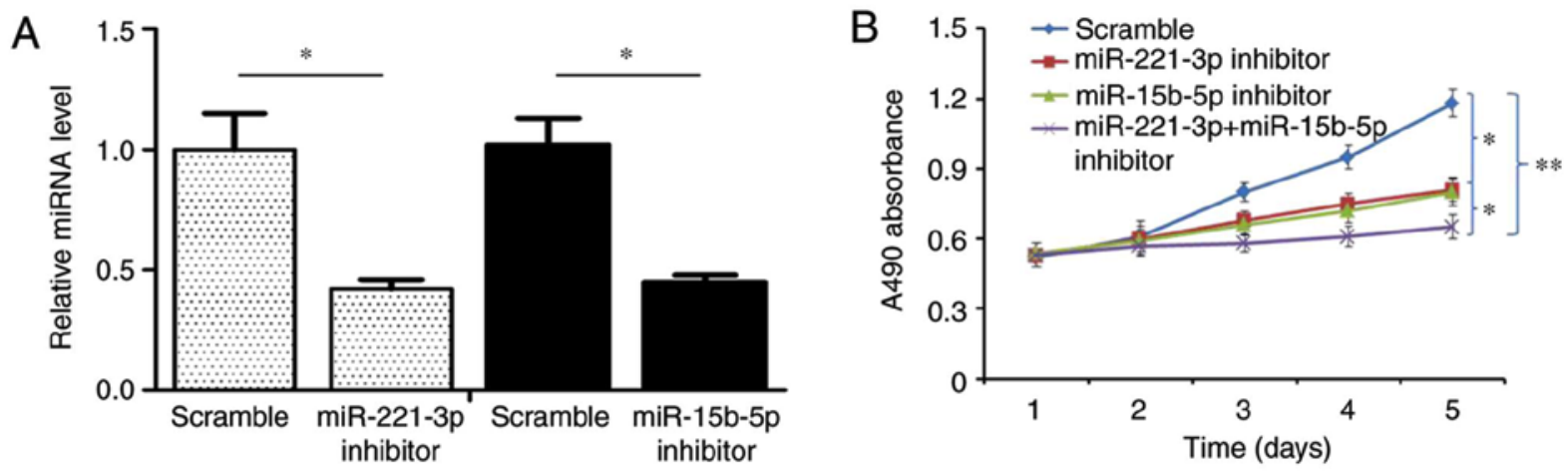

C
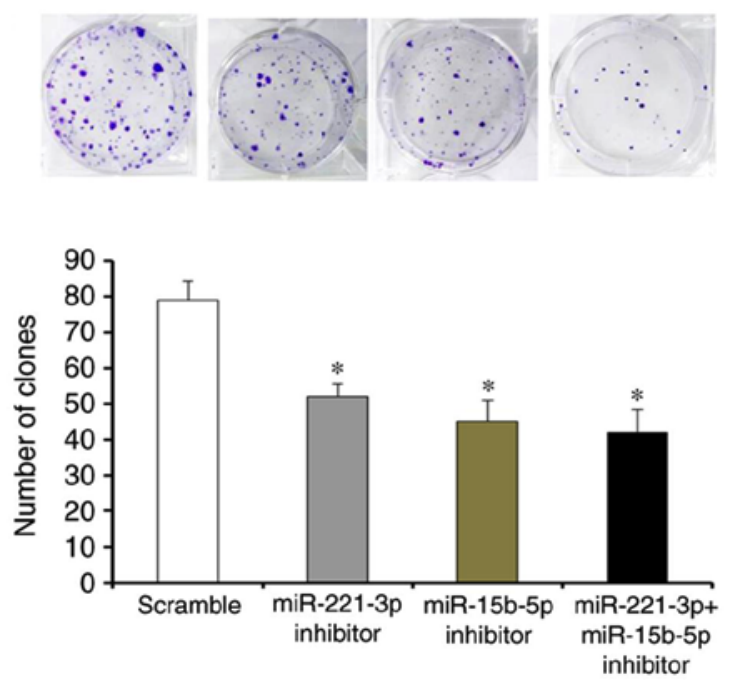
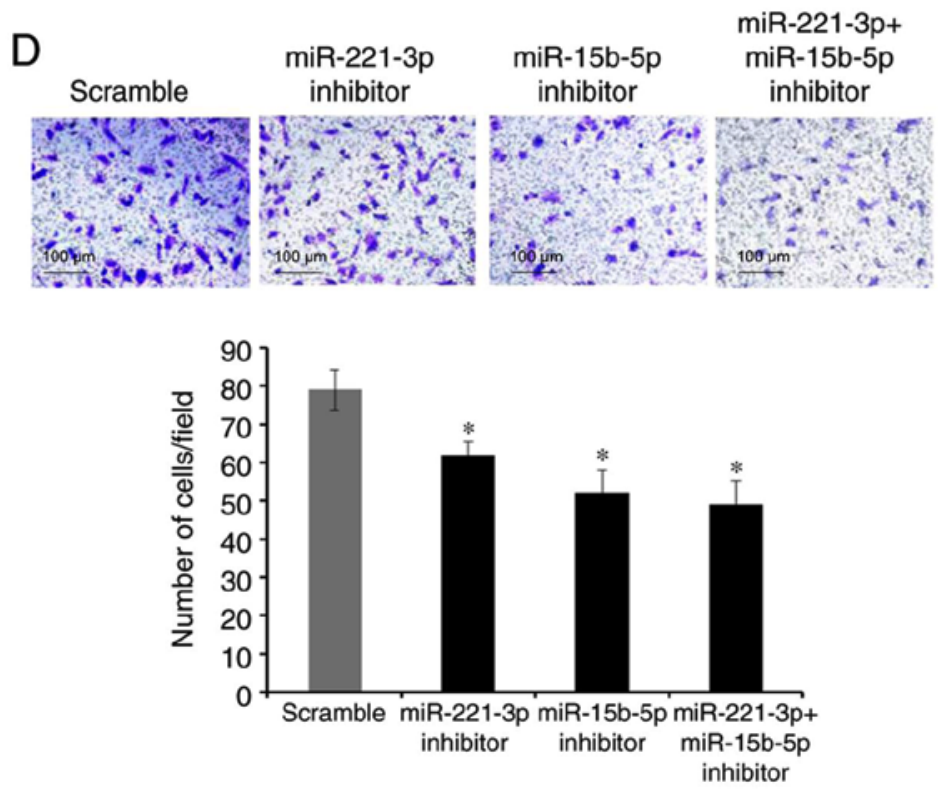

Figure 3. Knockdown of miR-221-3p and/or miR-15b-5p inhibits liver cancer cell proliferation, colony formation and invasion in vitro. (A) Quantitative PCR results demonstrated that the relative expression levels of miR-221-3p and miR-15b-5p were significantly lower in HepG2 cells transfected with miR-221-3p and miR-15b-5p inhibitors compared with the scrambled control. (B) Knockdown of miR-221-3p and/or miR-15b-5p significantly suppressed HepG2 cell proliferation compared with the control group. (C) Knockdown of miR-221-3p and/or miR-15b-5p significantly inhibited HepG2 colony formation compared with the control group. (D) Knockdown of miR-221-3p and/or miR-15b-5p significantly inhibited the invasive ability of HepG2 cells compared with the control group. ${ }^{*} \mathrm{P}<0.05$ and ${ }^{* *} \mathrm{P}<0.01$ vs. Scramble. miR, miRNA, microRNA.

a dual luciferase activity assay revealed that overexpression of miR-221-3p or miR-15b-5p inhibited the luciferase activity of the pGL3-Axin2-3'-UTR reporter, but not the pGL3-mut-Axin2-3'-UTR reporter $(\mathrm{P}<0.05$; Fig. $4 \mathrm{C}$ and D). Western blotting results demonstrated that Axin2 protein levels were significantly lower in liver cancer cells transfected with miR-221-3p or miR-15b-5p mimics $(\mathrm{P}<0.01$; Fig. 4E). Western blotting experiments were also performed to assess Axin2 expression in liver cancer tissues and corresponding adjacent non-cancerous tissues, and the results revealed that the protein expression level of Axin2 in HCC tissues was significantly lower compared with that in adjacent non-cancerous tissues $(\mathrm{P}<0.05$; Fig. $4 \mathrm{~F})$.

Axin2 is a common functional target of miR-221-3p and miR-15b-5p in liver cancer cells. To establish the roles of miR-221-3p and miR-15b-5p in the regulation of Axin2, HepG2 cells were transfected with si-Axin2 to knock down the endogenous expression of Axin2. The reduction and overexpression in Axin2 levels was determined by western blotting (Fig. 5A and B). Axin2 knockdown had a significant promotive effect on the proliferation and invasion of HepG2 cells, similar to the promotion of liver cancer cells by overexpression of miR-221-3p and miR-15b-5p (Fig. 5C and D). In HepG2 cells stably overexpressing miR-221-3p and miR-15b-5p, the restoration of Axin2 level reversed the promotive effects of miR-221-3p and miR-15b-5p in liver cancer cells (Fig. 5E and F). In addition, the results revealed that when knockdown of Axin2 was followed by transfection with miR-221-3p and miR-15b-5p inhibitor, Axin2 levels and invasive and colony formation abilities in liver cancer cells were partially restored (Fig. 6).

\section{Discussion}

Liver cancer is a primary liver tumor that ranks third in incidence among all gastrointestinal tumors globally $(1,16)$. Numerous studies have reported that miRNAs perform important functions in the development of liver cancer (17-21). Previous studies have demonstrated abnormal levels of 
A

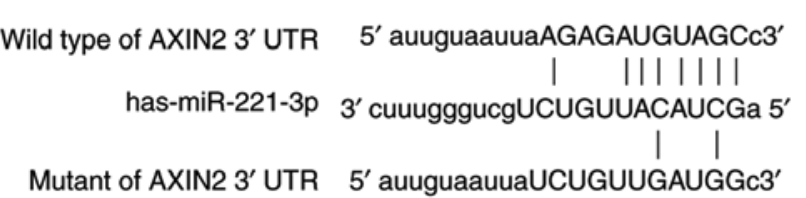

Wild type of AXIN2 3' UTR $5^{\prime}$ uuuuuuuuucCUAUGCUGCUc 3' Mas-miR-15b-5p 3 'acauuugguacuacACGACGAu5'

C

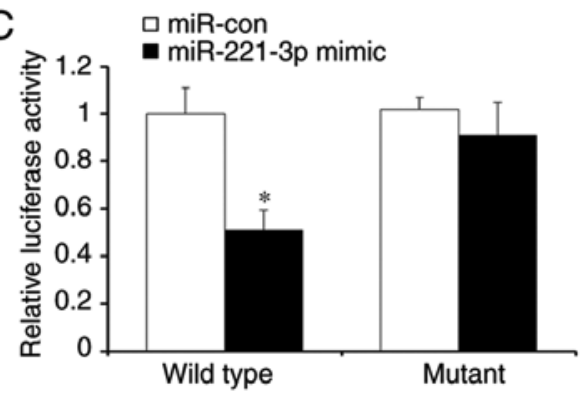

E
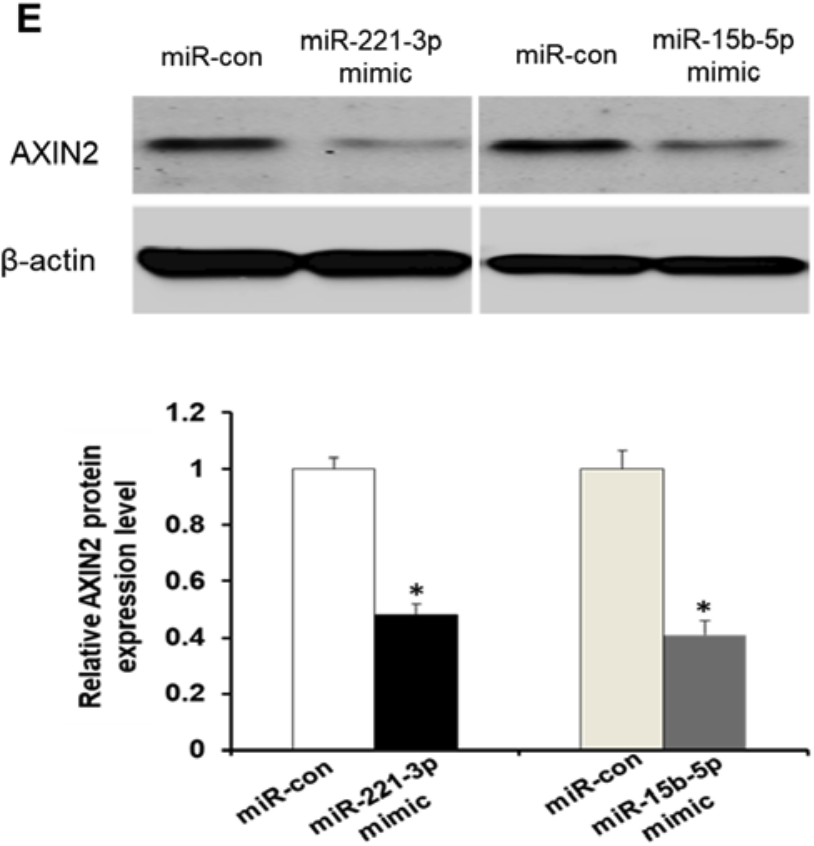

D

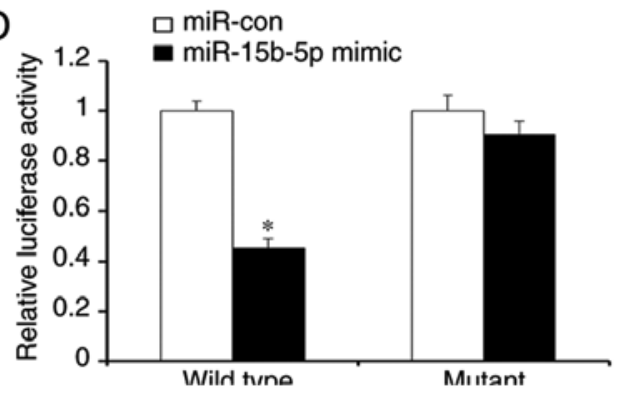

$\mathbf{F}$
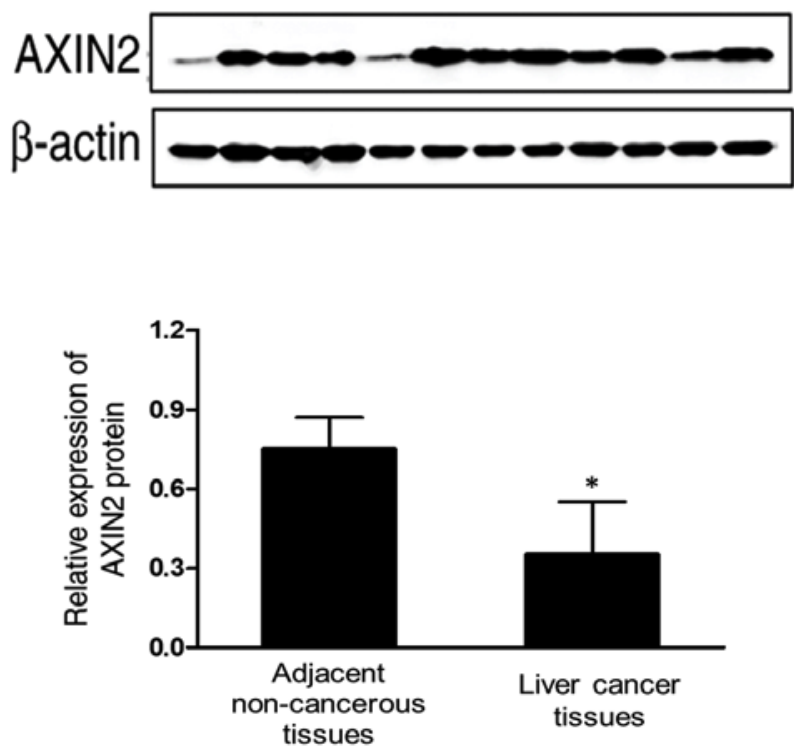

Figure 4. Axin2 is a direct common target of miR-221-3p and miR-15b-5p in liver cancer. (A) The schematic map of the pGL3-AXIN2-3'UTR and pGL3-AXIN2-mut-3'UTR binding site for miR-221-3p. (B) The schematic map of pGL3-AXIN2-3'UTR and pGL3-AXIN2-mut-3'UTR binding site for miR-15b-5p. (C) miR-221-3p targeted the AXIN2 wild type 3'UTR, but not the mutant. (D) miR-15b-5p targeted the wild type 3'UTR, but not the mutant. (E) Western blotting results demonstrated that overexpression of miR-221-3p or miR-15b-5p inhibited Axin2 protein expression in HepG2 cells. (F) Western blotting experiments were performed to detect Axin2 expression in liver cancer tissues and corresponding adjacent non-cancerous tissues; the results indicated the Axin2 protein expression in liver cancer tissues was significantly lower compared with that in adjacent non-cancerous tissues. "P<0.05 vs. miR-con or adjacent non-cancerous tissues. Axin2, axis inhibition protein 2; miR, microRNA; UTR, untranslated region; mut, mutant; miR-con, mimic control; T, tumor; $\mathrm{N}$, adjacent non-cancerous tissue; HCC, hepatocellular carcinoma.

miR-221 and miR-15b in liver cancer tissues, which affect the progression of liver cancer and exhibit oncogenic characteristics $(9,22-25)$. Although the mechanisms of action of these miRNAs in tumors are unclear, their importance is evident; miR-221 and miR-15b may be breakthrough targets for tumor prevention, diagnosis and treatment.

Recent studies have indicated that miR-221-3p and miR-15b-5p are involved in a number of types of cancer. For example, miR-221-3p exerts a tumor-suppressive role in epithelial ovarian cancer and directly targets ADP ribosylation factor 4 , which suggests that miR-221-3p may be a suitable candidate for the clinical prognosis and therapeutics for epithelial ovarian cancer (26); additionally, miRNA-221-3p serves an oncogenic role in gastric carcinoma by inhibiting PTEN expression (27), promotes the metastasis of gastric cancer through progestin and adipoQ receptor family member 3 and may be a biomarker of gastric cancer (28). The results of the present study indicated that miR-221-3p and miR-15b-5p were frequently upregulated in liver cancer tissues and cells and that high expression levels of miR-221-3p and miR-15b-5p were associated with TNM stage, infiltration and poor prognosis. In addition, overexpression of miR-221-3p and miR-15b-5p promoted liver cancer cell proliferation and invasion in vitro. These results indicated that miR-221-3p and miR-15b-5p may serve crucial roles in the occurrence of liver cancer and may promote its progression. 
A

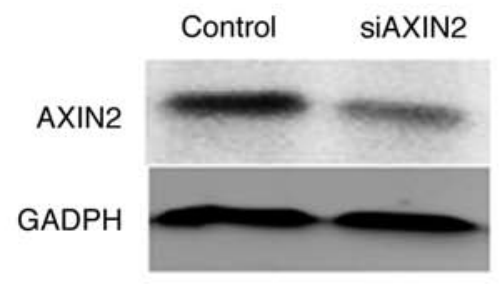

C

AXIN2
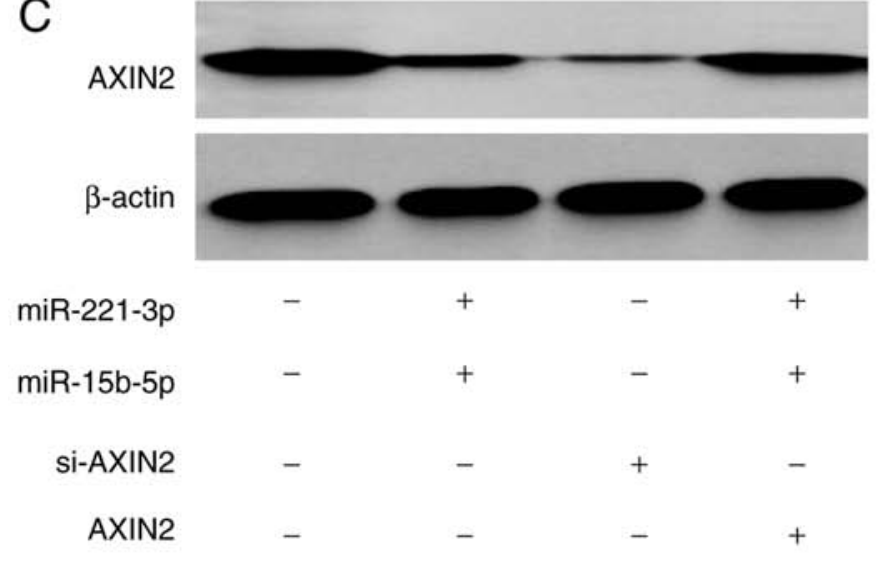

E

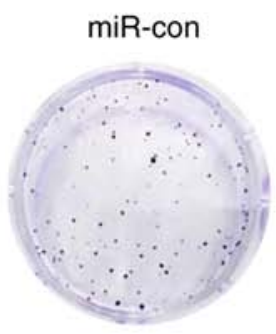

si-AXIN2

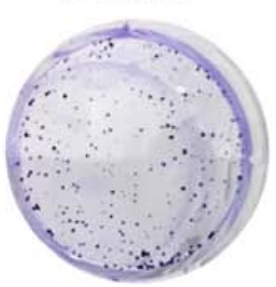

B

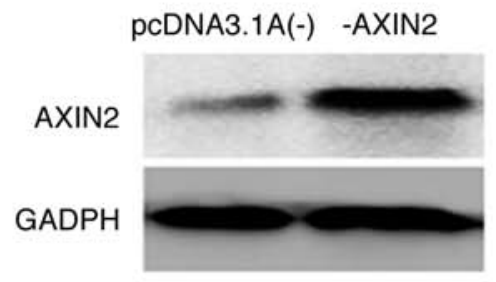

D

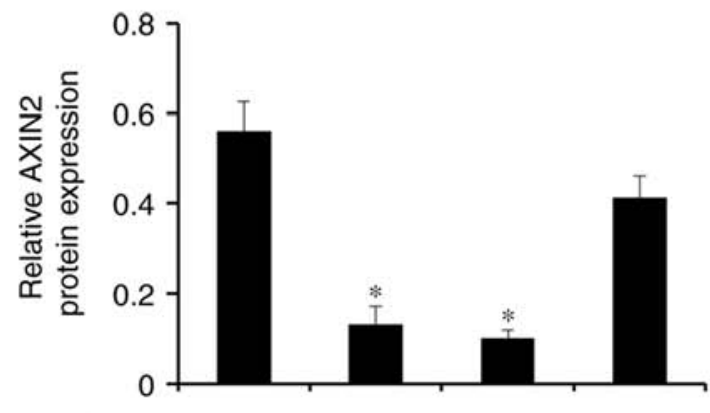

miR-221

miR-15b-5p

si-AXIN2

AXIN2

miR-con

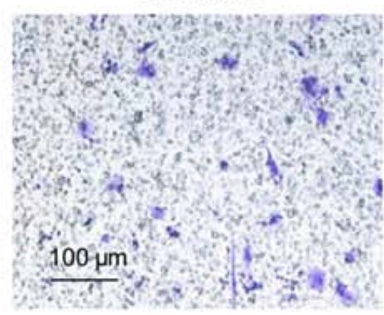

si-AXIN2

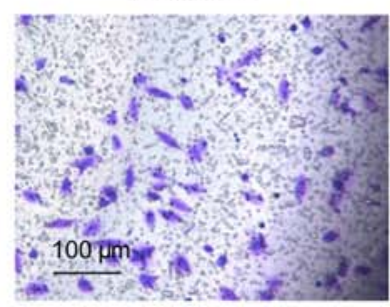

miR-221-3p+miR-15b-5p

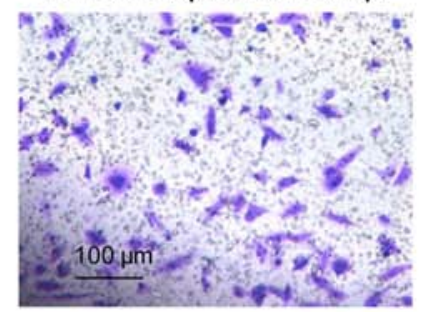

miR-221-3p/miR-15b-5p-AXIN2

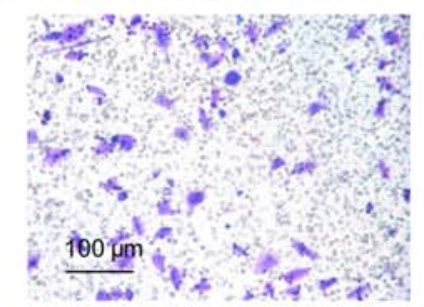

Figure 5. Axin2 is a common functional target of miR-221-3p and miR-15b-5p in liver cancer cells. (A) Western blot analysis of Axin2 expression in HepG2 cells transfected with si-AXIN2. Control cells were transfected with an empty plasmid. (B) Western blot analysis of Axin2 expression in HepG2 cells transfected with the Axin2-overexpressing plasmid pcDNA3.1A(-)-AXIN2 and the control plasmid pcDNA3.1A(-). (C and D) Western blot analysis was performed to assess the effects of ectopic miR-221-3p and miR-15b-5p expression, knockdown and reintroduction of Axin2 on the protein expression of Axin2 in liver cancer cells. (E) Colonies formed by liver cancer cells transfected with different plasmids. (F) Results of Transwell invasion assays in liver cancer cells transfected with different plasmids. "P<0.05 vs. control. miR, microRNA; con, control; Axin2, axis inhibition protein 2; si-AXIN2, small interfering RNA targeting Axin2; AXIN2, Axin2 overexpression plasmid.

Axin2 is an anti-oncogene that negatively regulates the canonical Wnt $/ \beta$-catenin signaling pathway, which includes a series of proteins such as the extracellular factor Wnt, T-cell factor and the frizzled transmembrane receptor. Axin2 is a scaffold protein involved in the degradation $\beta$-catenin complex when dephosphorylated (29). Mutations in the Axin2 gene have been identified in multiple types of human cancer, including tumors of the digestive tract and melanoma (30-33). The results of the present study predicted that Axin2 may be one of the target genes of miR-221 using bioinformatics tools, and luciferase and western blot assay results revealed that Axin2 was a direct target of miR-221. Knockdown of Axin2 promoted the proliferation of liver cancer cells, which further suggested that Axin2 may be a target of miR-221 in liver cancer.

In conclusion, the results of the present study demonstrated that miR-221-3p and miR-15b-5p were upregulated in liver cancer cells and were associated with TNM stage, 
A

C

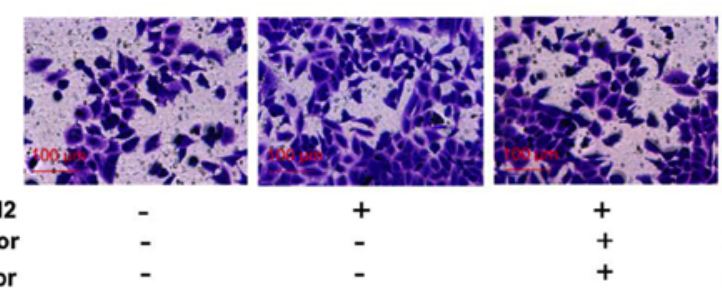
miR-15b-5p inhibitor

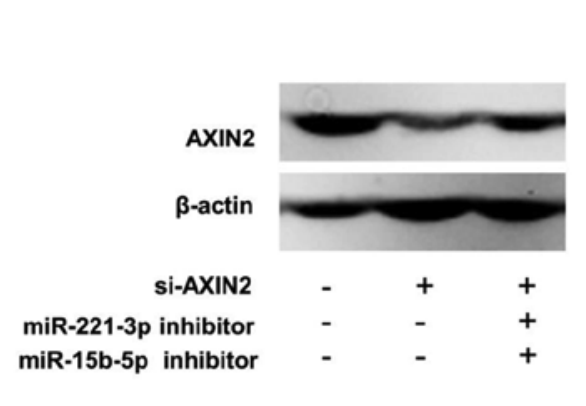

B

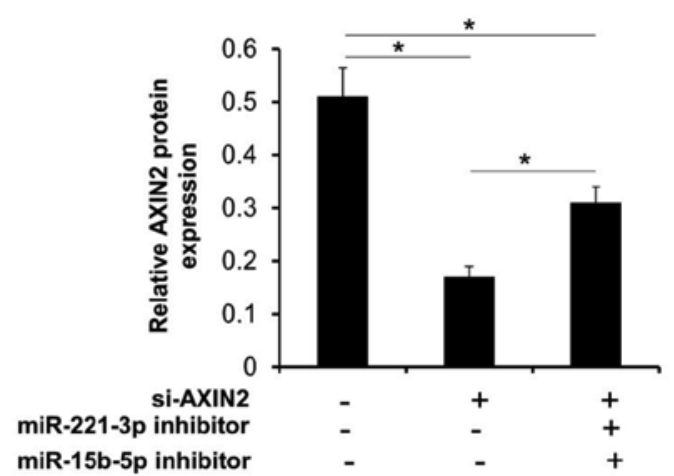

D
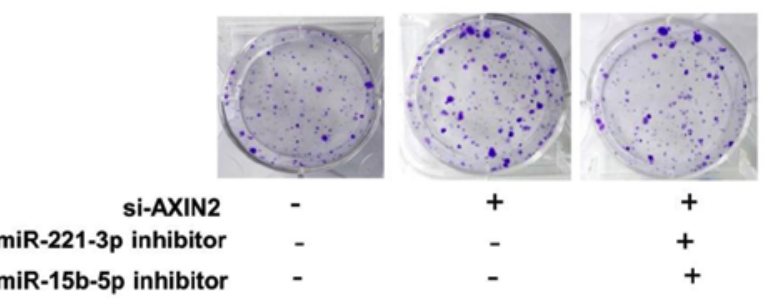
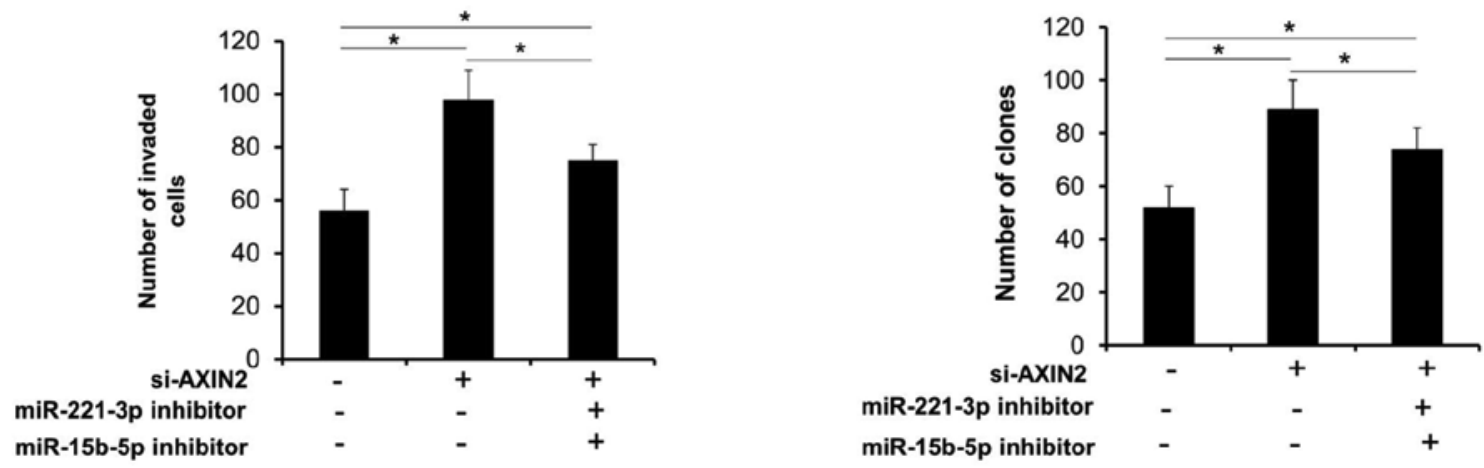

Figure 6. Axin2 is involved in miR-221-3p and miR-15b-5p-mediated cell proliferation and invasion in liver cancer. (A and B) Western blot analysis was performed to assess the expression of Axin2 following knockdown of Axin2 and/or inhibition of miR-221-3p and miR-15b-5p in liver cancer cells. (C and D) Following knockdown of Axin2 and/or inhibition of miR-221-3p and miR-15b-5p, (C) invasion and (D) colony formation were assessed in liver cancer cells. "P<0.05. miR, microRNA; Axin2, axis inhibition protein 2; si-AXIN2, small interfering RNA targeting Axin2.

tumor capsular infiltration and prognosis of patients with liver cancer. Overexpression of miR-221-3p and miR-15b-5p promoted liver cancer cell proliferation and invasion in vitro. In addition, Axin2 was identified as a direct and functional target of miR-221 in liver cancer cells. These results indicated that miR-221-3p and miR-15b-5p may be used as prognostic indicators for liver cancer. The miR-221-3p/miR-15b-5p-Axin2 axis may potentially serve as a therapeutic target for patients with liver cancer.

\section{Acknowledgements}

Not applicable.

\section{Funding}

The present study was supported by Nantong Science and Technology Project (grant nos. MS12018066 and MSZ18114).

\section{Availability of data and materials}

All data analyzed during the present study are included in this published article.

\section{Authors' contributions}

YD and XT conceived and designed the experiments. YD, NZ, SZ, XC, FL and XT performed all the experiments. NZ and XT wrote and revised the manuscript. All authors read and approved the final version of the manuscript.

\section{Ethics approval and consent to participate}

The present study was approved by the Research Ethics Committee of Linyi Central Hospital (Lin Yi, China). Written informed consent was obtained from all patients prior to enrolment. 


\section{Patient consent for publication}

Not applicable.

\section{Competing interests}

The authors declare that they have no competing interests.

\section{References}

1. Hernandez-Gea V, Turon F, Berzigotti A and Villanueva A: Management of small hepatocellular carcinoma in cirrhosis: Focus on portal hypertension. World J Gastroenterol 19: 1193-1199, 2013

2. Yang LY, Fang F, Ou DP, Wu W, Zeng ZJ and Wu F: Solitary large hepatocellular carcinoma: A specific subtype of hepatocellular carcinoma with good outcome after hepatic resection. Ann Surg 249: 118-123, 2009.

3. Liu X, Liao W, Yuan Q, Ou Y and Huang J: TTK activates Akt and promotes proliferation and migration of hepatocellular carcinoma cells. Oncotarget 6: 34309-34320, 2015.

4. Whittaker S, Marais R and Zhu AX: The role of signaling pathways in the development and treatment of hepatocellular carcinoma. Oncogene 29: 4989-5005, 2010.

5. Karavias D, Maroulis I, Papadaki H, Gogos C, Kakkos S, Karavias D and Bravou V: Overexpression of CDT1 is a predictor of poor survival in patients with hepatocellular carcinoma. J Gastrointest Surg 20: 568-579, 2016.

6. Chen K and Rajewsky N: The evolution of gene regulation by transcription factors and microRNAs. Nat Rev Genet 8: 93-103, 2007.

7. Ji J, Yamashita T and Wang XW: Wnt/beta-catenin signaling activates microRNA-181 expression in hepatocellular carcinoma Cell Biosci 1: 4, 2011.

8. Tomimaru Y, Eguchi H, Nagano H, Wada H, Kobayashi S, Marubashi S, Tanemura M, Tomokuni A, Takemasa I, Umeshita K, et al: Circulating microRNA-21 as a novel biomarker for hepatocellular carcinoma. J Hepatol 56: 167-175, 2012.

9. Rong M, Chen G and Dang Y: Increased miR-221 expression in hepatocellular carcinoma tissues and its role in enhancing cell growth and inhibiting apoptosis in vitro. BMC Cancer 13: 21, 2013.

10. Kim YK, Yu J, Han TS, Park SY, Namkoong B, Kim DH, Hur K, Yoo MW, Lee HJ, Yang HK and Kim VN: Functional links between clustered microRNAs: Suppression of cell-cycle inhibitors by microRNA clusters in gastric cancer. Nucleic Acids Res 37: 1672-1681, 2009.

11. Ye X, Bai W, Zhu H, Zhang X, Chen Y, Wang L,Yang A, Zhao J and Jia L: MiR-221 promotes trastuzumab-resistance and metastasis in HER2-positive breast cancers by targeting PTEN. BMB Rep 47: 268-273, 2014.

12. Chen LP, Zhang NN, Ren XQ, He J and Li $Y$ : miR-103/miR-195/miR-15b regulate SALL4 and inhibit proliferation and migration in glioma. Molecules 23: pii: E2938, 2018

13. Chen R, Sheng L, Zhang HJ, Ji M and Qian WQ: miR-15b-5p facilitates the tumorigenicity by targeting RECK and predicts tumour recurrence in prostate cancer. J Cell Mol Med 22: $1855-1863,2018$.

14. Hsu PK, Wu YC, Chou TY, Huang CS and Hsu WH: Comparison of the 6th and 7th editions of the American Joint Committee on Cancer tumor-node-metastasis staging system in patients with resected esophageal carcinoma. Ann Thorac Surg 89: 1024-1031, 2010.

15. Livak KJ and Schmittgen TD: Analysis of relative gene expression data using real-time quantitative PCR and the 2(-Delta Delta C(T)) method. Methods 25: 402-408, 2001.

16. Jemal A, Bray F, Center MM, Ferlay J, Ward E and Forman D: Global cancer statistics. CA Cancer J Clin 61: 69-90, 2011.

17. Kapodistrias N, Bobori C and Theocharopoulou G: MiR-140-3p downregulation in association with PDL-1 overexpression in many cancers: A review from the literature using predictive bioinformatics tools. Adv Exp Med Biol 988: 225-233, 2017 (In German).
18. Wang G, Dong F, Xu Z, Sharma S, Hu X, Chen D, Zhang L, Zhang J and Dong Q: MicroRNA profile in HBV-induced infection and hepatocellular carcinoma. BMC Cancer 17: 805, 2017.

19. Wen Y, Han J, Chen J, Dong J, Xia Y, Liu J, Jiang Y, Dai J, Lu J, Jin $\mathrm{G}$, et al: Plasma miRNAs as early biomarkers for detecting hepatocellular carcinoma. Int J Cancer 137: 1679-1690, 2015.

20. Morgul MH, Klunk S, Anastasiadou Z, Gauger U, Dietel C, Reutzel-Selke A, Felgendref P, Hau HM, Tautenhahn HM, Schmuck RB, et al: Diagnosis of $\mathrm{HCC}$ for patients with cirrhosis using miRNA profiles of the tumor-surrounding tissue-A statistical model based on stepwise penalized logistic regression. Exp Mol Pathol 101: 165-171, 2016.

21. Lu L, Guo D, Chen X, Xiong W, Jie S and Li H: Abnormal miRNAs targeting chromosome open reading frame genes were enriched in microvesicles derived from the circulation of HCC. Biochem Genet 54: 120-133, 2016.

22. Fornari F, Gramantieri L, Ferracin M, Veronese A, Sabbioni S, Calin GA, Grazi GL, Giovannini C, Croce CM, Bolondi L and Negrini M: MiR-221 controls CDKN1C/p57 and CDKN1B/p27 expression in human hepatocellular carcinoma. Oncogene 27: 5651-5661, 2008

23. Park JK, Kogure T, Nuovo GJ, Jiang J, He L, Kim JH, Phelps MA, Papenfuss TL, Croce CM, Patel T and Schmittgen TD: miR-221 silencing blocks hepatocellular carcinoma and promotes survival. Cancer Res 71: 7608-7616, 2011.

24. Duan Z, Gao Y, Shen J, Choy E, Cote G, Harmon D, Bernstein K, Lozano-Calderon S, Mankin H and Hornicek FJ: miR-15b modulates multidrug resistance in human osteosarcoma in vitro and in vivo. Mol Oncol 11: 151-166, 2017.

25. Ahmad P, Sana J, Slavik M, Gurin D, Radova L, Gablo NA, Kazda T, Smilek P, Horakova Z, Gal B, et al: MicroRNA-15b-5p predicts locoregional relapse in head and neck carcinoma patients treated with intensity-modulated radiotherapy. Cancer Genomics Proteomics 16: 139-146, 2019.

26. Wu Q, Ren X, Zhang Y, Fu X, Li Y, Peng Y, Xiao Q, Li T, Ouyang C, Hu Y, et al: MiR-221-3p targets ARF4 and inhibits the proliferation and migration of epithelial ovarian cancer cells. Biochem Biophys Res Commun 497: 1162-1170, 2018.

27. Shi J, Zhang Y, Jin N, Li Y, Wu S and Xu L: MicroRNA-221-3p plays an oncogenic role in gastric carcinoma by inhibiting PTEN expression. Oncol Res 25: 523-536, 2017.

28. Zhao C,Li Y, Chen G, Wang F, Shen Z and Zhou R: Overexpression of miR-15b-5p promotes gastric cancer metastasis by regulating PAQR3. Oncol Rep 38: 352-358, 2017.

29. Clevers $\mathrm{H}$ and Nusse $\mathrm{R}$ : Wnt $/ \beta$-catenin signaling and disease. Cell 149: 1192-1205, 2012.

30. Lammi L, Arte S, Somer M, Jarvinen H, Lahermo P, Thesleff I, Pirinen S and Nieminen P: Mutations in AXIN2 cause familial tooth agenesis and predispose to colorectal cancer. Am J Hum Genet 74: 1043-1050, 2004.

31. Rivera B, Perea J, Sanchez E, Villapún M, Sánchez-Tomé E, Mercadillo F, Robledo M, Benítez J and Urioste M: A novel AXIN2 germline variant associated with attenuated FAP without signs of oligondontia or ectodermal dysplasia. Eur J Hum Genet 22: 423-426, 2014.

32. Castiglia D, Bernardini S, Alvino E, Pagani E, De Luca N, Falcinelli S, Pacchiarotti A, Bonmassar E, Zambruno G and D'Atri S: Concomitant activation of Wnt pathway and loss of mismatch repair function in human melanoma. Genes Chromosomes Cancer 47: 614-624, 2008

33. Kim MS, Kim SS, Ahn CH, Yoo NJ and Lee SH: Frameshift mutations of Wnt pathway genes AXIN2 and TCF7L2 in gastric carcinomas with high microsatellite instability. Hum Pathol 40: 58-64, 2009.

(i) $(-)$ This work is licensed under a Creative Commons Attribution-NonCommercial-NoDerivatives 4.0 International (CC BY-NC-ND 4.0) License. 